\title{
Effects of statins on the prognosis of local and locally advanced renal cell carcinoma following nephrectomy
}

\author{
SEUNG-KWON CHOI, GYEONG EUN MIN, SEUNG HYUN JEON, HYUNG-LAE LEE, \\ SUNG-GOO CHANG and KOO HAN YOO
}

Department of Urology, School of Medicine, Kyung Hee University, Seoul 134-727, Republic of Korea

Received October 6, 2012; Accepted December 3, 2012

DOI: $10.3892 / \mathrm{mco} .2012 .55$

\begin{abstract}
The anti-angiogenic properties of statins, widely used for the treatment of hyperlipidemia, have recently been reported. The aim of this study was to investigate the effects of use of statins for the management of post-operative recurrence and progression of renal cell carcinoma (RCC). Of 306 patients diagnosed with kidney cancer between January, 2006 and June, 2012, 115 patients who showed no distant metastasis and had undergone radical or partial nephrectomy were selected. These patients were divided into a group that took statins and a group that did not. The effects of statins on the progression and recurrence of renal cancer were retrospectively analyzed. The demographics of the statins group showed that there were more males $(\mathrm{P}=0.039)$ and that they were of older age [mean age, $65.24( \pm 6.82)$ vs. $58.95( \pm 12.33)$ years; $\mathrm{P}=0.039]$ and of higher body mass index (BMI) [26.17 ( \pm 2.96$)$ vs. 24.24 $( \pm 3.35), P=0.017]$. Recurrence-free survival in the two groups showed 59.44 and 66.72 months for the statin and non-statin groups, respectively, with no statistically significant difference $(\mathrm{P}=0.586)$. Progression-free survival also showed no statistically significant difference between the two groups $(\mathrm{P}=0.307)$. Results of the multivariate analysis using Cox's regression model revealed that gender, age and BMI had no significance as prognostic factors for the recurrence and progression of renal cancer ( $P>0.05)$. Findings of the present study demonstrated that statins do not have a marked effect on the recurrence and progression of renal cancer. Therefore, further investigation using a larger patient group should be conducted for future statistical analysis.
\end{abstract}

\section{Introduction}

Statins are cholesterol-lowering agents that act by inhibiting the enzyme 3-hydroxy-3-methylglutaryl coenzyme A (HMG-CoA) reductase. In addition, statins are also widely

Correspondence to: Dr Koo Han Yoo, Department of Urology, School of Medicine, Kyung Hee University, 149 Sangil-Dong, Gangdong-Gu, Seoul 134-727, Republic of Korea

E-mail: yookoohan@khu.ac.kr

Key words: carcinoma, renal cell, hydroxymethylglutaryl-CoA reductase inhibitors, prognosis used in the primary as well as secondary prevention of cardiovascular diseases, especially hypercholesterolemia $(1,2)$. They reduce long-term cerebrovascular events, particularly after an initial coronary event (3). Beyond their lipid-lowering effects, statins have also been shown to exhibit anti-inflammatory (4) and antineoplastic (5) activities. Although the precise mechanisms by which these activities occur are unknown, they seem to correlate with the inhibitory effects of statins on the mevalonate pathway. By inhibiting $\mathrm{HMG}-\mathrm{CoA}$ reductase and controlling mevalonate synthesis, statins affect cholesterol biosynthesis and control the production of several additional major products, including dolichol, geranyl pyro- and farnesyl pyrophosphate (4-6). This process may be relevant for the induction of antitumor responses, as these agents have effects on various additional cell functions, including DNA synthesis, cell cycle progression and apoptosis (5). Consistent with these observations, statins have been shown to exert regulatory effects on cell cycle progression and to induce apoptosis of malignant cells $(5,7)$.

Statins have also been recognized for their activity against various types of cancer. The use of statins as anticancer agents is based on pre-clinical evidence of their anti-proliferative, pro-apoptotic, anti-invasive and radiosensitizing properties (8). Statins exhibit in vitro anti-neoplastic activities against a variety of malignant cells of diverse origin $(7,9)$. Progress has been noted with regard to the effects of statins against various types of malignancy. However, information is scarce on the effects of these agents against renal cell carcinoma (RCC) (7). The aim of this study was to investigate the effect of statins on the recurrence and progression of RCC.

\section{Patients and methods}

Patients. A retrospective analysis was conducted on patients diagnosed with RCC who presented at our hospital between January 1, 2006 and June 30, 2012. Of the 306 RCC patients, a total of 115 patients who had radical or partial nephrectomy (open or laparoscopic) for non-distant metastatic (any TN0-1M0) RCC were included in the present study. No patient had a known history of von Hippel-Lindau disease, contralateral nephrectomy for RCC or synchronous bilateral RCC. Informed consent was obtained from all patients.

Patients were divided into the statin and non-statin groups. Patients taking a statin following the diagnosis of RCC were 
Table I. Clinical characteristics and correlation between the two groups.

\begin{tabular}{|c|c|c|c|c|}
\hline Characteristics & $\begin{array}{l}\text { Statin } \\
\text { group }\end{array}$ & $\begin{array}{l}\text { Non-statin } \\
\text { group }\end{array}$ & Total & P-value \\
\hline Gender & & & & 0.039 \\
\hline Male & 63 & 9 & 72 & \\
\hline Female & 31 & 12 & 43 & \\
\hline Age (years) & $58.95 \pm 12.33$ & $65.24 \pm 6.82$ & & 0.002 \\
\hline BMI & $24.24 \pm 3.35$ & $26.17 \pm 2.96$ & & 0.017 \\
\hline Cancer type & & & & 0.71 \\
\hline Clear cell & 78 & 18 & 96 & \\
\hline Papillary & 4 & 0 & 4 & \\
\hline Chromophobe & 9 & 2 & 11 & \\
\hline Collecting duct & 2 & 0 & 2 & \\
\hline Sarcomatoid & 1 & 1 & 2 & \\
\hline T stage & & & & 0.935 \\
\hline T1a & 47 & 14 & 61 & \\
\hline T1b & 25 & 4 & 29 & \\
\hline $\mathrm{T} 2 \mathrm{a}$ & 5 & 1 & 6 & \\
\hline $\mathrm{T} 2 \mathrm{~b}$ & 5 & 1 & 6 & \\
\hline $\mathrm{T} 3 \mathrm{a}$ & 9 & 1 & 10 & \\
\hline $\mathrm{T} 3 \mathrm{~b}$ & 2 & 0 & 2 & \\
\hline $\mathrm{T} 4$ & 1 & 0 & 1 & \\
\hline N stage & & & & 0.628 \\
\hline No & 91 & 21 & 112 & \\
\hline N1 & 3 & 0 & 3 & \\
\hline
\end{tabular}

BMI, body mass index.

excluded from the statin group. The dose and type of statin used and compliance were not factored in the analysis.

Statistical analysis. Statistical analysis was performed with SPSS ver. 16.0 (SPSS, Chicago, IL, USA). The correlation between gender, pathologic characteristics including Fuhrman grade, cell type and tumor, node, metastasis (TNM) stage of the two groups were analyzed using the Chi-square test. The correlation between age and body mass index (BMI) of the two groups was analyzed using the Student's t-test. Multivariate analysis was performed using Cox's regression model to determine the effect of confounding variables. The Kaplan-Meier survival test was used to determine the association between recurrence and progression of the two groups. $\mathrm{P}<0.05$ was considered to indicate a statistically significant difference.

\section{Results}

Of the total of 115 patients [male, $72(63 \%)$ and female, $43(37 \%)]$, the statin group comprised $21(18 \%)$ patients taking statins, while the non-statin group comprised 94 (82\%)patients not taking statins. Patient clinical characteristics are shown in Table I. The clear cell type was the most common RCC type, comprising $96(83 \%)$ patients. Other types included the chromophobe, papillary, collecting duct and sarcomatoid

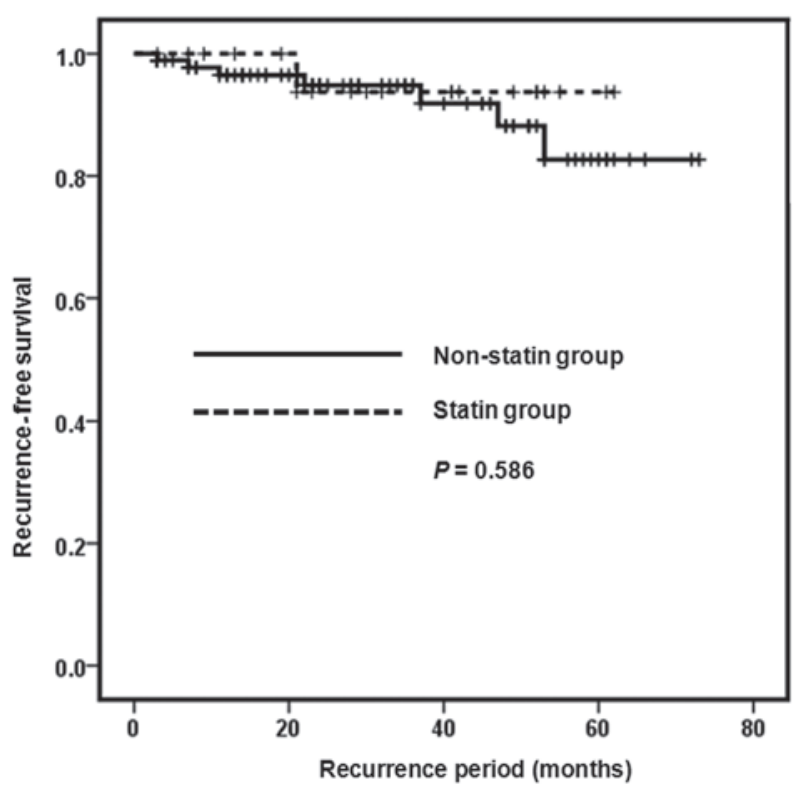

Figure 1. Recurrence-free survival curve of the patients.

comprising $11(10 \%), 4(3 \%), 2(2 \%)$ and $2(2 \%)$ patients, respectively. T1a was the most common $\mathrm{T}$ stage, followed by T1b, comprising $61(53 \%)$ and 29 (25\%) patients, respectively. N0 was identified in $112(97 \%)$ and N1 in $3(3 \%)$ patients. During the follow-up, $8(7 \%)$ and $4(3 \%)$ patients exhibited recurrence and progressive disease, respectively.

Male gender was identified significantly more frequently in the statin group, compared with the non-statin group $(\mathrm{P}=0.039)$. The RCC type and TNM stage did not vary in the two groups. Mean age was higher in the statin compared with the non-statin group $[65.24( \pm 6.82)$ and $58.95( \pm 12.33)$, respectively] $(\mathrm{P}=0.002)$. Mean $\mathrm{BMI}$ was higher in the statin compared with the non-statin group [24.24 $( \pm 3.35)$ and 26.17 $( \pm 2.96)$, respectively] $(\mathrm{P}=0.026)$ (Table I). When assessed using the Kaplan-Meier survival analysis, the recurrence and progression-free survival rate of the two groups showed no statistically significant difference $(\mathrm{P}=0.586$ and $\mathrm{P}=0.307)$ (Figs. 1 and 2). The effects of gender, age, BMI and statins on recurrence and progression were examined, using Cox's regression model. However, the effects were not statistically significant (Table II).

\section{Discussion}

Statins have a lowering effect of low-density lipoprotein (LDL) cholesterol by inhibiting HMG-CoA reductase. Mevalonate is a limiting factor in hepatic cholesterol biosynthesis as it is an essential precursor (6). Thus, statins were prescribed for hypercholesterolemic patients. Statins were also administered to patients with cardiovascular diseases, including myocardial infarction and atrial fibrillation (2,10-12).

The underlying mechanisms of the lowering effect of statins on the incidence of cerebrovascular disease have yet to be elucidated. However, several studies suggest that the metabolites of statins have certain regulatory effects on the endothelial, platelet and leukocyte function. Their function is regulated by G-proteins, such as Rho, Rac and Ras, and 
Table II. Results of the effects of gender, age, BMI and statins on recurrence and progression. ${ }^{\mathrm{a}}$

\begin{tabular}{lcc}
\hline Characteristics & Exp (B) & P-value \\
\hline Gender & 1.322 & 0.770 \\
Age & 0.968 & 0.260 \\
BMI & 1.065 & 0.572 \\
Statins & 0.787 & 0.846 \\
\hline
\end{tabular}

${ }^{a}$ Multivariate analysis using Cox's regression model. BMI, body mass index. Exp (B), exponentiation of the B coefficient.

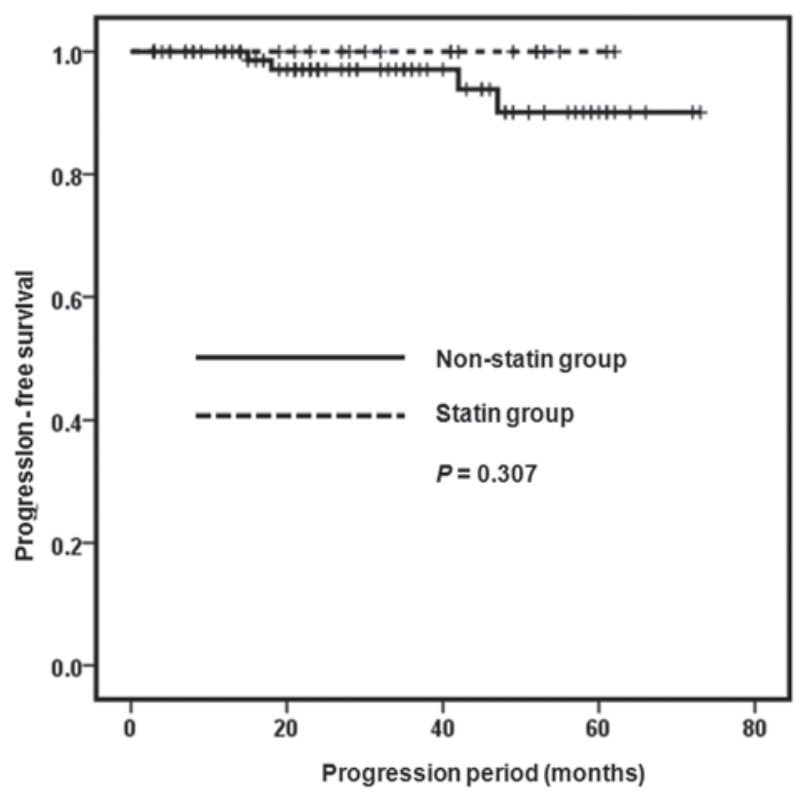

Figure 2. Progression-free survival curve of the patients.

intermediates of HMG-CoA reductase are essential for their post-translational modification $(13,14)$. Additionally, statins modulate vascular remodeling by inhibiting extracellular matrix metalloproteinases and transcription factors, such as nuclear factor- $\kappa B$ (14). Statins have been shown to attenuate vasoconstriction by increasing endothelial nitric oxide activity (15). Thus, they have pleiotropic cardiovascular and anti-atherosclerotic effects, including improvement of endothelial function, reduction of free radical formation, stabilization of plaques and inhibition of endothelial inflammatory reactions, which contribute to other potential benefits for patients at risk of cardiovascular disease, regardless of cholesterol levels $(8,16)$.

The mechanisms of these antitumor effects have yet to be fully elucidated. Findings of previous studies suggest that statins have antitumor effects by inhibiting tumor cell growth, angiogenesis and induction of apoptosis. Statins block tumor cell growth in vitro and in vivo, thus inhibiting dolichol, geranyl pyro- and farnesyl pyrophosphate production, Rasand Rho-mediated cell proliferation and the stabilization of the cell cycle kinase inhibitors p21 and p27 (17). Although statins have been reported to stimulate and inhibit angiogenesis, they inhibit capillary tube formation and decrease vascular endothelial growth factor release (18). Statins exert pro-apoptotic properties in a variety of tumor cells, by which they upregulate pro-apoptotic proteins, decrease anti-apoptotic proteins and activate certain types of caspases $(19,20)$. Statins impair the metastatic potential of tumor cells by inhibiting cell migration, attachment to the extracellular matrix and invasion of basement membrane (21).

However, clinical data regarding the antitumor effects of statins are conflicting. In certain studies, statins apparently have pro-carcinogenic effects. Coogan et al (22) conducted a case control study on 1,132 women with breast cancer and 1,009 men with prostate cancer. Controls included 1,331 women and 1,387 men who presented with conditions unrelated to statin use. The odds ratio (OR) for breast cancer among statin users was 1.5 (95\% CI, 1.0-2.3), largely accounted for by an OR of 1.8 (95\% CI, 0.9-3.6) among cases with carcinoma in situ. Among the invasive cases, the OR was 1.2 (95\% CI, 0.7-2.0). The OR for prostate cancer overall was 1.2 (95\% CI, 0.8-1.7) and 1.4 (95\% CI, 0.7-2.5). A retrospective analysis of 2,463 women showed an increased risk of breast cancer among statin users of 5.2\% (11).

Several other studies maintain that statins have an antitumor effect. Poynter et al (23) conducted a population-based case-control study on 1,953 patients with colorectal cancer and 2,015 controls. Their results showed that the use of statins for at least five years (vs. the non-use of statins) was associated with a significantly reduced relative risk of colorectal cancer (OR=0.50; 95\% CI, 0.40-0.63). Khurana et al (8) performed a retrospective nested case-control study of 500,000 veteran patients and observed that statin use is associated with a $44 \%$ risk reduction of RCC. Liu et al (1) performed a retrospective analysis of data of two US cohorts, and confirmed the beneficial effect of statin use on the risk of RCC. Authors of that study suggested that the use of statins was associated with a reduced risk of RCC in women. The association was statistically significant in women with no history of hypertension. In men, a reduced risk was identified only among individuals with no history of hypertension.

Findings of the present study have shown that statins have no significant effects on recurrence and progression in patients that have undergone radical nephrectomy following diagnosis with RCC. A randomized double-blind trial of mortality and incidence of cancer during a 10-year follow-up of the Scandinavian Simvastatin Survival Study has shown no difference in mortality from and incidence of cancer between the simvastatin and placebo groups (24). Several studies have found a similar relative risk for cancer development $(25,26)$, the results of which are partly consistent with our findings.

At present, available studies $(1,8,23)$ have focused on the antitumor effect of statins and the risk of cancers, which varies from our study. In this study, we focused on the effects of statins on recurrence and progression of already diagnosed RCC. To the best of our knowledge, studies on the effects of statins on recurrence and progression-free survival of diagnosed RCC are rare.

Our study has certain limitations. It is a retrospective study that may have some biases. Due to the small population size, our results are difficult to generalize to other RCC patients. Comorbidities of the patients were not analyzed 
in this study, nor were the dose, type of statin used or the compliance.

In conclusion, statins do not have a marked effect on recurrence and progression-free survival of non-distant metastatic RCC patients. Additional, well-designed studies on large populations and with a long-term follow up are necessary to assess the effects on statins of these patients.

\section{References}

1. Liu W, Choueiri TK and Cho E: Statin use and the risk of renal cell carcinoma in 2 prospective US cohorts. Cancer: 118, 797-803, 2012.

2. Heart Protection Study Collaborative Group: MRC/BHF Heart Protection Study of cholesterol lowering with simvastatin in 20,536 high-risk individuals: a randomised placebo-controlled trial. Lancet 360: 7-22, 2002.

3. Hebert PR, Gaziano JM, Chan KS and Hennekens CH: Cholesterol lowering with statin drugs, risk of stroke, and total mortality. An overview of randomized trials. JAMA 278: 313-321, 1997.

4. Forrester JS and Libby P: The inflammation hypothesis and its potential relevance to statin therapy. Am J Cardiol 99: 732-738, 2007.

5. Demierre MF, Higgins PD, Gruber SB, Hawk E and Lippman SM: Statins and cancer prevention. Nat Rev Cancer 5: 930-942, 2005.

6. Goldstein JL and Brown MS: Regulation of the mevalonate pathway. Nature 343: 425-430, 1990.

7. Woodard J, Sassano A, Hay N and Platanias LC: Statin-dependent suppression of the Akt/mammalian target of rapamycin signaling cascade and programmed cell death 4 up-regulation in renal cell carcinoma. Clin Cancer Res 14: 4640-4649, 2008.

8. Khurana V, Caldito G and Ankem M: Statins might reduce risk of renal cell carcinoma in humans: case-control study of 500,000 veterans. Urology 71: 118-122, 2008.

9. Sivaprasad U, Abbas T and Dutta A: Differential efficacy of 3-hydroxy-3-methylglutaryl CoA reductase inhibitors on the cell cycle of prostate cancer cells. Mol Cancer Ther 5: 2310-2316, 2006.

10. Shepherd J, Cobbe SM, Ford I, et al: Prevention of coronary heart disease with pravastatin in men with hypercholesterolemia. West of Scotland Coronary Prevention Study Group. N Engl J Med 333: 1301-1307, 1995.

11. Sacks FM, Pfeffer MA, Moye LA, et al: The effect of pravastatin on coronary events after myocardial infarction in patients with average cholesterol levels. Cholesterol and Recurrent Events Trial investigators. N Engl J Med 335: 1001-1009, 1996.

12. Young-Xu Y, Jabbour S, Goldberg R, et al: Usefulness of statin drugs in protecting against atrial fibrillation in patients with coronary artery disease. Am J Cardiol 92: 1379-1383, 2003.
13. Cipollone F, Fazia M, Iezzi A, et al: Suppression of the functionally coupled cyclooxygenase-2/prostaglandin E synthase as a basis of simvastatin-dependent plaque stabilization in humans. Circulation 107: 1479-1485, 2003.

14. Krupski WC, Layug EL, Reilly LM, et al: Comparison of cardiac morbidity between aortic and infrainguinal operations. Study of Perioperative Ischemia (SPI) Research Group. J Vasc Surg 15: 354-365, 1992

15. Bax L, Mali WP, Buskens E, et al: The benefit of STent placement and blood pressure and lipid-lowering for the prevention of progression of renal dysfunction caused by Atherosclerotic ostial stenosis of the Renal artery. The STAR-study: rationale and study design. J Nephrol 16: 807-812, 2003.

16. Farmer JA: Pleiotropic effects of statins. Curr Atheroscler Rep 2: 208-217, 2000

17. Rao S, Lowe M, Herliczek TW, et al: Lovastatin mediated G1 arrest in normal and tumor breast cells is through inhibition of CDK2 activity and redistribution of p21 and p27, independent of p53. Oncogene 17: 2393-2402, 1998.

18. Vincent L, Chen W, Hong L, et al: Inhibition of endothelial cell migration by cerivastatin, an HMG-CoA reductase inhibitor: contribution to its anti-angiogenic effect. FEBS Lett 495: 159-166, 2001

19. Cafforio P, Dammacco F, Gernone A, et al: Statins activate the mitochondrial pathway of apoptosis in human lymphoblasts and myeloma cells. Carcinogenesis 26: 883-891, 2005.

20. Marcelli M, Cunningham GR, Haidacher SJ, et al: Caspase-7 is activated during lovastatin-induced apoptosis of the prostate cancer cell line LNCaP. Cancer Res 58: 76-83, 1998.

21. Hindler K, Cleeland CS, Rivera E and Collard CD: The role of statins in cancer therapy. Oncologist 11: 306-315, 2006.

22. Coogan PF, Rosenberg L, Palmer JR, et al: Statin use and the risk of breast and prostate cancer. Epidemiology 13: 262-267, 2002.

23. Poynter JN, Gruber SB, Higgins PD, et al: Statins and the risk of colorectal cancer. N Engl J Med 352: 2184-2192, 2005.

24. Strandberg TE, Pyorala K, Cook TJ, et al: Mortality and incidence of cancer during 10-year follow-up of the Scandinavian Simvastatin Survival Study (4S). Lancet 364: 771-777, 2004.

25. ALLHAT Officers and Coordinators for the ALLHAT Collaborative Research Group: Major outcomes in moderately hypercholesterolemic, hypertensive patients randomized to pravastatin vs. usual care: the Antihypertensive and Lipid-Lowering Treatment to Prevent Heart Attack Trial (ALLHAT-LLT). JAMA 288: 2998-3007, 2002.

26. Simes RJ, Marschner IC, Hunt D, et al: Relationship between lipid levels and clinical outcomes in the Long-term Intervention with Pravastatin in Ischemic Disease (LIPID) Trial: to what extent is the reduction in coronary events with pravastatin explained by on-study lipid levels? Circulation 105: 1162-1169, 2002. 